\title{
Cloud Assisted P2P Media Streaming for Bandwidth Constrained Mobile Subscribers
}

\author{
Xin Jin, Yu-Kwong Kwok \\ Dept. of Electrical \& Electronic Engg, The University of Hong Kong, Pokfulam, Hong Kong, China
}

\begin{abstract}
Multimedia streaming applications have disruptively occupied bandwidth in wireline Internet, yet today's fledging mobile media streaming still poses many challenges in efficient content distribution due to the form of mobile devices. At the same time, cloud computing is gaining power as a promising technology to transform IT industry and many eminent enterprises are developing their own cloud infrastructures. However, the lack of applications hinders clouds' large-scale implementation. In this paper, we envision a cloud-assisted power-efficient mobile P2P media streaming architecture that addresses the weakness of today's wireless access technologies. Clouds are responsible for storage and computing demanding tasks, and mobile devices colocating with each other share bandwidth and cooperatively stream media content to distribute the load. We first model interactions among mobile devices as a coalition game, and then discuss the optimal chunk retrieval scheduling. Finally, we draw on realistic mobile phone data and utilize an ARIMA model for colocation duration prediction among mobile devices.
\end{abstract}

Index Terms - cooperative mobile media streaming, cloud, p2p, coalition game, ARIMA

\section{INTRODUCTION}

Future mobile devices may turn out to be mobile supercomputers as the integration of GPS, video camera, etc., with a decent battery lifetime [1]. With the growing penetration of powerful mobile devices, mobile applications are emerging as a significant new application form in the next generation Internet, among which media streaming may disruptively occupy the bandwidth of mobile devices just like in wireline Internet. However, current wireless Internet access technologies can only provide the choice between high average bitrate (e.g., WiFi or WiMAX) and good coverage area (e.g., cellular networks), but cannot guarantee both at the same time [2]. At the same time, the limitations of contrainted computational capacity, occasional connectivity loss and limited power supply are threatening the full blossom of this new application form. In this paper, we focus our discussions on a cloud assisted cooperative media streaming in scenarios that mobile devices cannot individually achieve a reasonable streaming rate due to bandwidth limitation.

As an exceptional assistance, clouds provide us with a platform to perform storage and computing demanding tasks remotely. Cloud computing - the integration of Software as a Service (SaaS), Platform as a Service (PaaS) and Infrastructure as a Service (IaaS) [3], [4] - has the potential to mitigate frustrations faced by mobile devices: mobile devices send task requests to clouds, which perform corresponding operations (e.g., resource discovery) and return retrieved media content or other information. Based on this storage and computing model, mobile devices may save a substantial amount of power and also overcome the defects of limited local computational and storage resources. In addition, this reduces communication overheads between mobile devices and content providers. In this paper, we refer to cloud as any entity that can provide a remote service for mobile devices.

We still need a communication model to solve problems of constrained bandwidth and occasional connectivity loss. We assume that each mobile device is equipped with a low bitrate wireless Internet access interface (e.g., cellular Internet interface) and a high speed local wireless network interface (e.g., Bluetooth interface). Consequences of occasional connectivity losses can be easily mitigated utilizing techniques of prefetching and caching. Recent research about significant temporal periodicity and spacial regularity of human mobility [5] - people spend most of their time in a few locations, such as home and workplace - offers us a chance to overcome constainted bandwidth: communication cooperation can be formed by people from the same community or with similar human behaviors. That is, colocating mobile devices (peers) may share wireless Internet bandwidth with each other to obtain higher streaming rate by distributing load among themselves through underlying sharing links (i.e., high speed local wireless links) [2].

The purpose of this paper is to obtain a in-depth understanding of cooperative mobile media streaming through coalition game analysis, through which we obtain that system information is beneficial to cooperative streaming formulation. People move across environments, which poses higher churn rates for mobile media streaming. This again points out the necessity to obtain system information about peer movement, following which we propose a colocation prediction algorithm. Targetting cooperative mobile media streaming formulation, our contributions in this paper are three-fold:

- To better understand interactions among peers cooperating with each other for media streaming, we model the cooperative mobile streaming problem as a coalition game. We derive that energy supply and system information about peer mobility are the foremost factors for coalition formulation.

- To minimize energy consumpution, we propose a cloud assisted system architecture for cooperative media streaming and analyze the system under a generic concept of content providers and underlying sharing links among peers.

- To acquire peer mobility information, clouds collect colocation statistics, predict the colocation durations between peers, allocate peer pairs with highest predicted colo- 
cation durations for cooperative streaming, henceforth limit peer churns to the minimum and achieve optimal chunk scheduling. Considering the limited capacity of some current mobile devices, we assume that peers scan colocation peers periodically via short-range wireless network interfaces (e.g., Bluetooth, increasingly being left switched on [6]).

The rest of this paper proceeds as follows. We begin by detailing the background of recent advances in mobile cooperative streaming (Section II). In Section III, colaborations among peers are modeled as coalition games, from which we derive some system design implications. In Section IV, colocation statistics analysis are presented, before Section V summerizes our discussions and sketches our furture plans.

\section{BACKGROUND}

\section{A. Content Sharing}

In [7] by McNamara et al., a media sharing mechanism is designed to utilize colocation among commuters and renders it possible to share content with each other in urban transport. Analyses about how to handle colocation and share content with peers potentially colocating with each other are conducted. By selecting peers with the longest average colocation duration, this mechanism ensures that each peer downloads an intact file from another peer with higher probability. However, this paper is restricted to opportunistic communication through short-range wireless interfaces in urban transport without Internet connections and colocation duration prediction may not be satisfiable.

\section{B. Mobile P2P Streaming}

A realtime $\mathrm{p} 2 \mathrm{p}$ mobile streaming system is proposed in [8] by Peltotalo et al., demonstrating the feasibility of $\mathrm{P} 2 \mathrm{P}$ live streaming in mobile networks. Xie et al. [9] illustrate their open source project of $\mathrm{p} 2 \mathrm{p}$ live media streaming for handheld devices, and demonstrate the low bandwidth and limited computation capacity in a handheld device. Akkanen et al. [10] show the technical feasibility to use portable devices to actively broadcast media streams. Stiemerling et al. [2] propose a P2P-TV system that enables mobile users to cooperatively retrieve media chunks in resource constrained mobile environments.

\section{Modeling Cooperative P2P Streaming}

In this section, we first propose our system architecture and formulate cooperative mobile streaming problem, followed by a coalition game analysis.

\section{A. System Architecture}

As depicted in Figure 1, in our system architecture, we move computation demanding and peer administration tasks onto clouds, considering the power, bandwidth, computation, and storage limitations of mobile devices. For example, peers are assisted with clouds for content discovery from content providers, namely retrieval of content destination information, especially when streaming from an existing P2P overlay.

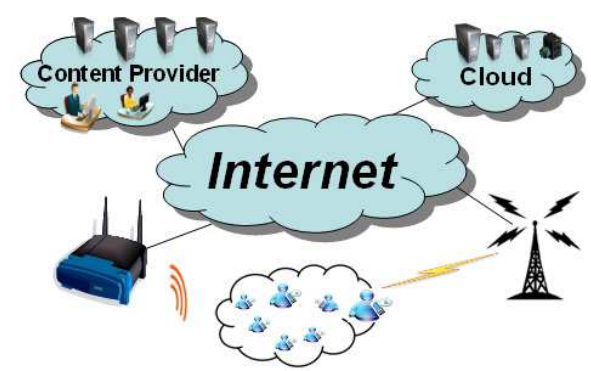

Figure 1. System Architecture.

Clouds also assist in the formulation of cooperative streaming via colocation statistics. For clarity, we denote by $R\left(u_{i}\right)$ the content retrieved by peer $u_{i}$, and $u_{i}$ is in the proximity of $u_{j}$. If $R\left(u_{i}\right)=R\left(u_{j}\right)$, they may cooperate with each other to download content and make best use of bandwidth; otherwise, the one with spare bandwidth can potentially benefact the other through social ties.

\section{B. Problem Formulation and Local Streaming Cooperation}

Due to bandwidth scarcity, a single peer with wireless Internet connections may not possess enough bandwidth to maintain a satisfactory streaming quality. In reality, colocation peers may cooperate with each other when they are interested in the same streaming content or "familiar" with each other via a built-in social network. Therefore, content and bandwidth sharing among peers is desirable for the streaming satisfaction of peers: each peer streams one portion of the media and exchange them with each other. To obtain a long-term streaming quality, peers contribute their bandwidth endowments in a cooperative manner and form a coalition. One peer joins a coalition only if it obtains extra benefits, otherwise it will keep out of this coalition. Thus, a coalition game theoretic analysis is called for.

\section{Game Formulation}

The proposed collaborative streaming problem can be modeled as a coalition game given by $\langle\mathcal{N}, v\rangle$, where function $v$ associates with every nonempty subset $S$ of $\mathcal{N}$ a real number $v(S)$ - the worth of $S$-independent with the behavior of the coalition $N / S$. A coalition $S$ is a subset of the entire peer set $\mathcal{N}=\{0,1, \ldots,|\mathcal{N}|\}$. Peers in a coalition are willing to cooperate with each other, and herein the worth of $S$ is called the characteristic function.

Each peer $i$ in the collaborative streaming system cares about its average power consumption $c_{i}$ and average streaming rate $s_{i}$ in the period of streaming, both of which are determined by cooperations among peers and vary w.r.t. $S$. Denote by $B_{i}^{w}$ bandwidth of its wireless Internet access interface, and $B_{i}^{t}$ bandwidth of its underlying local wireless network interface. Power constraint is specified as follow: $c_{i} \leq C_{i}$, where $C_{i}$ depends on the specification of $i$ 's power supply; the streaming constraint is

$$
s_{i}=s \leq B_{i}^{w}+B_{i}^{t} \text { and } s_{i} \leq B_{i}^{w}+\sum_{j \in \mathcal{T}} s_{j i},
$$


where $\mathcal{T}$ is the set of current peers helping $i$ and $s_{j i}$ is the streaming rate that $i$ obtains from $j$. We assume that communication for local cooperation is equipped with high capacity links and peers within a coalition share the same streaming quality. To this end, peer $i$ 's streaming rate merely depends on its residential coalition; henceforth, we drop the subscript $i$ in the streaming rate notation.

The utility function of peers within a coalition $S \in \mathcal{N}$ must capture benefits earned from media streaming with the sacrifice of power consumption. For this purpose, peer $i$ 's utility function $v_{i}(S)$ must be an increasing function of streaming rate $s$ and a decreasing function of power consumption $c_{i}$. Before discussing characteristic function $v(S)$, the utility for node $i$ in collaborative streaming is given by

$$
v_{i}(S)=g_{i}(s)-f_{i}\left(c_{i}\right),
$$

where $g_{i}(s)$ indicates the utility obtained, and $f_{i}\left(c_{i}\right)$ the cost function of power consumption. Streaming quality obtained by each peer should be non-decreasing with respect to each peer's bandwidth endowment. For ease of analysis, we introduce effort strength $\eta_{i}$ to denote the bandwidth endowment of peer $i$, and $\mu_{i}$, the interest level of peer $i$ to the current streaming content in coalition $S$. In reality, peers value and are willing to enjoy the streaming media iff interest levels are higher than some interest level threshold $\mu^{*}$. Hence, $g_{i}(s)$ is practically defined as an increasing and twice differentiable concave function [11]:

$$
g_{i}(s)=g(s) \triangleq \begin{cases}0 & \mu_{i} \leq \mu^{*} \\ \log \left(1+\sum_{i \in S} \eta_{i}\right) & \mu_{i} \geq \mu^{*},\end{cases}
$$

which is independent with specific peers. Any well suited cost function $f_{i}\left(c_{i}\right)$ should be nondecreasing with an upper bound tolerable power consumption. Following [12], a cost function satisfying these requirements is log barrier penalty function given by

$$
f_{i}\left(c_{i}\right) \triangleq \begin{cases}-t a_{i}{ }^{2} \log \left(1-\left(\frac{c_{i}}{a_{i}}\right)^{2}\right) & c_{i}<a_{i} \\ +\infty & c_{i} \geq a_{i},\end{cases}
$$

where $t>0$ is a scale factor and $a_{i}$ is a power consumption constraint.

The worth of a coalition $S$ is equal to the streaming quality of each peer in the coalition, taking away cost due to power consumption. The characteristic function $v(S)$ is properly given by

$$
v(S)=g(s)-\sum_{i \in S} f_{i}\left(c_{i}\right)
$$

According to [11], a general coalition game $\langle N, X, V,(\succeq$ )$\left._{i \in N}\right\rangle$ is defined as: $X=\mathbb{R}^{N}, V(S)=\left\{x \in \mathbb{R}^{N}: \sum_{i \in S} x_{i}=\right.$ $v(S)$ and $x_{j}=0$ if $\left.j \in N \backslash S\right\}$ for each coalition $S$, and $x \succeq_{i} y$ if and only if $x_{i} \geq y_{i}$. In the following, we provide definitions of transferable utility and core, and subsequently discuss the corresponding properties of our proposed coalition game.

DEFINITION 1. A coalition game is a game with transferable utility if $v(S)$ may be distributed in any way among the members of $S$. Otherwise, the coalition game is a game without transferable utility, and each coalition $S$ is characterized by an arbitrary set $\mathrm{V}(\mathrm{S})$ of consequences.

PROPOSITION 1. The proposed coalition streaming game model is a game without transferable utility.

Proof. The characteristic function of a coalition $S$ is given by Eqn. (1). Since the streaming quality of each peer is also the streaming quality of the coalition, utility obtained from streaming quality is equal for each peer in the coalition. Although cost due to power consumption can be divided among peers within a coalition, we still have $v(S) \neq \sum_{i \in S} v_{i}(S)$. Therefore, the proposed coalition game has no transferable utility.

DEFINITION 2. The core of the coalition game $\langle N, X, V,(\succeq$ )$\left._{i \in N}\right\rangle$ is the set of all $x \in V(N)$ for which there is no coalition $S$ and $y \in V(S)$ so that $y \succ_{i} x$ for all $i \in S$.

The coalition needs to bring benefits to peers willing to cooperate. The core of the coalition game refers to a stable coalition so that no coalition enjoys the capacity to upset current cooperative coalition, and no peer holds incentive to leave it. The following theorem discusses whether a grand coalition will form in our proposed coalition game.

THEOREM 1. For our proposed coalition game $\langle N, X, V,(\succeq$ )$\left._{i \in N}\right\rangle$, the grand coalitions of all peers with similar content interest does NOT always form; rather, disjoint independent coalitions will emerge.

Proof. First, any peer $i$ with interest level $\mu_{i}<\mu^{*}$ will get negative utility if it joins the coalition, so this kind of peers have no incentive to join coalition. Secondly, peers without sufficient power supply will not join the coalition due to high cost. Finally, for peers with enough power supply and strong interest, we assume $v_{i}(S)=v(S)=g(s)$, and consider the following two cases. If peer $i$ has little or even no opportunity to form local sharing link with other peers in a coalition $S$ either spatially or temporally, it is not reasonable for it to stay in coalition $S$. Also, information incompleteness due to peer mobility, underlying sharing link, etc. does not guarantee that peers always form a grand coalition.

In a nutshell, we have a coalition game without transferable utility. In general, coalition game based problems seek to derive algorithms for forming coalitions among peers. Since coalition formulation has been a topic of high interest in game theory, it is not difficult to find a suitable coalition formulation algorithm. However, in this paper, we focus on the system design implications of our proposed coalition game. In reality, we assume that there exists a well-defined protocol in underlying sharing links.

\section{System Design Implications}

Coalition game model presents us with illuminating system design implications:

1) Iff one peer can contribute to and obtain streaming benefits from an existing coalition, it will be included 
in the coalition.

2) Energy deficiency of mobile devices will definitely render them noncooperative, in which case peers consider power consumption more urgent than the leisure of media entertainment.

3) System information about peer mobility is beneficial to the formulation of collaborative coalitions.

In this paper, we assume that, due to limited bandwidth, mobile devices equipped with decent energy supplies cannot obtain satisfactory streaming quality without cooperative streaming. For this purpose, we propose a cloud assisted social network architecture to minimize power consumption and maximize system information about peer mobility.

\section{Colocation Statistics}

In this section, we discuss streaming coordination and colocation statistics among peers.

\section{A. Optimal Chunk Retrieval Coordination Problem}

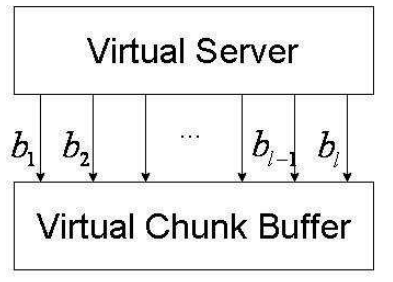

(a) Chunk Retrieval Coordination

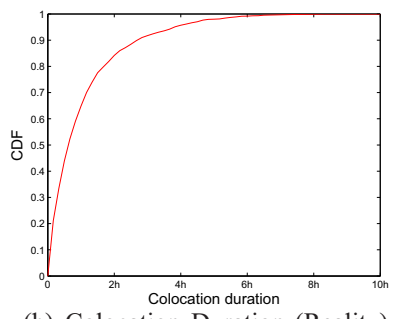

(b) Colocation Duration (Reality)

Figure 2. Chunk Retrieval Coordination and Colocation Duration.

Here, we show our modeling efforts for chunk retrieval coordination in a coalition. For analysis clarity, as in [2], we collapse the group of $N$ nodes into a single node with $N$ access links and a virtual chunk buffer; at the same time, we shield details about content providers and clouds as a virtual server as depicted in figure 2(a). Peers cooperate with each other for media streaming. For each coalition, we assume that there is a super peer with corresponding capacities-coalition coordinator-responsible for chunk retrieval coordination; otherwise, clouds fall back as the coalition cooradinator.

At time period $t, a_{i}=\mathbb{1}\{$ link $i$ is up and in the coalition $\}$, $x_{j}^{i}=\mathbb{1}\{$ the coalition uses link $i$ to download chunk $j\}$, and $\mathbb{1}\{\cdot\}$ is the indicator function, i.e., $\mathbb{1}(x)=1$ if $x$ is true, and $\mathbb{1}(x)=0$ otherwise. $b_{i}$ denotes the bandwidth of link $i$ at period $t$. The optimal chunk retrieval coordination favors links with higher bandwidth and shows priority to chunks more urgent for retrieval. Therefore, we formulate the coalition utility to retrieve chunk $j$ with playback time $T_{j}$ via link $i$ as

$$
U_{j}^{i}=\alpha U_{L}\left(a_{i} b_{i}\right)+(1-\alpha) U_{C}\left(T_{j}-t\right),
$$

where $\alpha>0, U_{L}(\cdot)$ is non-decreasing and $U_{C}(\cdot)$ is nonincreasing.

For each time period, the chunk retrieval coordination is equivalent to the assignment of tuples $\langle i, j\rangle$. Thus, we call $\langle i, j, t\rangle$ a retrieval coordination in period $t$ for chunk $j$. Denote by $b_{j}^{i}$ the bandwidth utilized by $i$ for the retrieval of chunk $j$.
Our target is to maximize the total utility of the coalition in the entire media streaming process:

$$
\max \sum_{j} U_{j}^{i} x_{j}^{i}
$$

subject to

$$
b_{j}^{i} \leq b_{i}, t \leq T_{j}
$$

If we possess the perfect information about inbound links, such an optimization problem can be trivially solved in the following fashion. At time $t$, coordinator $c$ selects the link with highest bandwidth to retrieve the most urgent chunk, i.e. the chunk with $\min \left(T_{j}-t\right)$. In the event that there is still available inbound links, c assigns the second urgent chunk to the corresponding link similarly. This process repeats until no available links are left, or all chunks for streaming are retrieved.

However, information incompleteness of available inbound links and link bandwidth fluctuations renders optimal chunk retrieval coordination extremely difficult. For illustration, suppose that we totally have three links for media retrieval at time period $t$ - link 1, 2, and 3 can retrieve 1 chunk, 2 chunks, and 2 chunks respectively. Now, if the coalition need 5 chunks, each link retrieves chunks with their own capacity. However, in the event that the coalition need 7 chunks, due to incompleteness of information about whether each link is still available for chunk retrieval in the next time period, it is difficult for the coordinator to determine how to schedule the chunk retrieval. Thus, we have the following proposition.

PROPOSITION 2. For cooperative mobile streaming, the optimal chunk retrieval problem is equivalent to colocation prediction among peers.

\section{B. Active Prediction of Colocation Duration and Social Net- work for Local Cooperation}

In this section, we draw on one dataset from the MIT Reality Mining Project [13], referred to as Reality, for colocation statistics. As shown in figure 2(b), we obtain CDF of colocation duration. The fact that most peers colocate with each other for a short duration illustrates the necessity of colocation duration prediction for cooperative streaming formulation to minimize peer churns and increase peer utility. After colocation duration prediction, we justify the need to build a social network based on peer colocation statistics as a platform for incentive mechanism design to motivate peers in proximity but without similar streaming interests to cooperate with each other.

Colocation Duration Prediction. We leverage clouds for colocation statistics. As stated in [7], we need to consider the temporal and spacial regularity, and cyclic variations of human mobility [5], which lead to the necessity of personalized profiles to record trajectory histories of peers on clouds. We utilize an ARIMA model for colocation duration prediction. We practically assume that peers are location-aware in terms of peer proximity and utilize Bluetooth scanning as a illustrative scenario in the following description. 
Clouds update profiles in the following fashion. Each peer periodically discovers peers in reach and reports to clouds. Clouds maintain a personalized profile for each peer, including colocation durations with another peer. However, clouds do not need to maintain a catalog for all colocations of each peer it meets in daily life; rather, clouds only keep a record of peers with colocation duration larger than a threshold (e.g., 100 seconds in the following analysis). When clouds receive a peer's report about the coincidence of a stranger (i.e., a peer not in its personalized profile), clouds check colocation duration at this time. If larger than the threshold, it adds it into the familiar profile. Otherwise, it adds this stranger to a stranger profile. If the peer meet this stranger frequently, it adds this stranger to the useless familiar profile to avoid redundant handling. In this way, we may detect the arrivals and departures of peers in $u$ 's proximity.

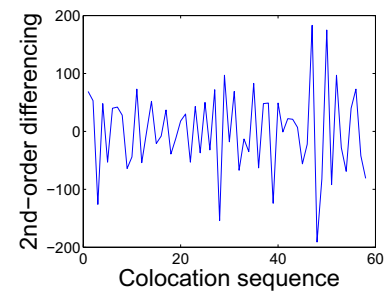

(a)
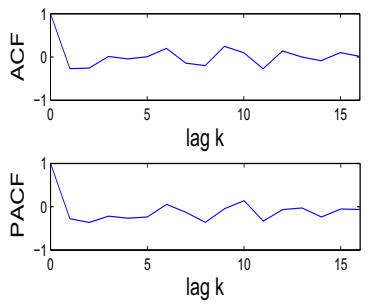

(b)
Figure 3. ARIMA Model Identification.

As the colocation duration time series $x_{t}$ is generally nonstationary (i.e., a time series contains a systematic change), the autoregressive integrated moving average model, $\operatorname{ARIMA}(p, d, q)$ - a standard linear predictor to tackle nonstationary time series - is utilized for colocation duration prediction [14]. ARIMA(p,d,q) involves two steps: the decision of $p, d, q$ (model identification) and the estimation of $p+q$ weight coefficients (model estimation). We derive an $\operatorname{ARIMA}(0,2,1)$ model in that the 2nd-order differencing is zero mean, values of autocorrelation (ACF) for longer lags tend to be zero and partial autocorrelation (PACF) tails off (figure 3). Therefore,

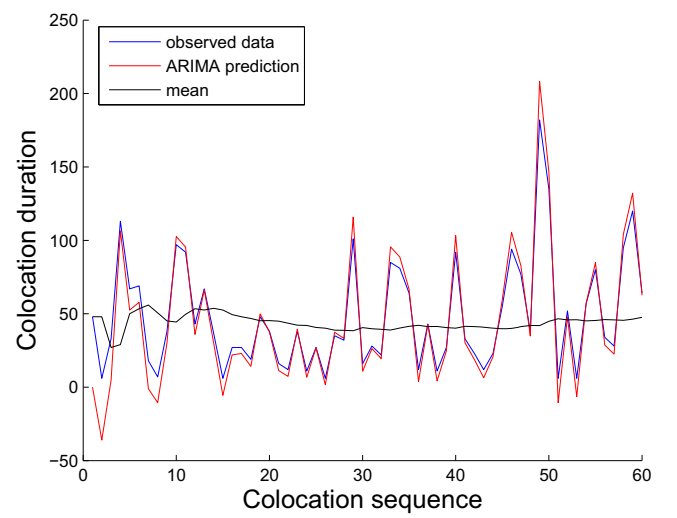

Figure 4. Colocation Duration Prediction with $\operatorname{ARIMA}(0,1,1)$. we can derive the prediction function

$$
\widehat{x}_{t+1}=2 x_{t}-x_{t-1}-\epsilon\left(x_{t}-\widehat{x}_{t}\right),
$$

where $\widehat{x}_{t}$ is the predicted time series and $\epsilon$ is the coefficient for the random error $x_{t}-\widehat{x}_{t}$, trained with colocation duration statistics. As depicted in figure 4 , the observed data $x_{t}$ is the colocation duration time series between two peers. We notice that the ARIMA prediction time seires $\widehat{x}_{t}$ is obviously much better than the mean method utilized in [7].

Social Network for Incentive Mechanism Design. Based on the above peer colocation statistics, clouds may build an social network infrastructure to encourage cooperations among colocation peers, even when they do not share the same content interest. When peer $u$ is in need of bandwidth, it reports current peers in reach, and clouds predict the remaining colocation duration with each discovered peer. Then, clouds send requests to peers with longest prediction colocation duration in the social network to help $u$. In reality, social network builds bonds among mobile users and potentially guarantees a platform for the formulation of an exchange economy and a well-designed infrastructure for effortless coalition formulation.

\section{CONClusion}

In this paper, we propose a cloud-assisted system architecture for media streaming in mobile Internet to minimize power consumption of mobile devices. We show our modeling efforts for cooperative streaming utilizing coalition games and optimal chunk retrieval coordination. Further, we predict colocation durations between peers with the aid of an ARIMA model, which is very important for cooperative streaming formulation. The predicted time series show better accurracy than normally used mean method. System implementation remains for further study.

\section{REFERENCES}

[1] T. Austin, D. Blaauw, S. Mahlke, T. Mudge, C. Chakrabarti, and W. Wolf, "Mobile supercomputers," Computer, pp. 81-83, 2004.

[2] M. Stiemerling and S. Kiesel, "A system for peer-to-peer video streaming in resource constrained mobile environments," in U-NET'09, 2009.

[3] M. Armbrust, A. Fox, R. Griffith, A. Joseph, R. Katz, A. Konwinski, G. Lee, D. Patterson, A. Rabkin, I. Stoica, and M. Zaharia, "Above the clouds: A berkeley view of cloud computing," EECS Department, University of California, Berkeley, Tech. Rep. UCB/EECS-2009-28, 2009.

[4] L. Wang, J. Tao, M. Kunze, A. Castellanos, D. Kramer, and W. Karl, "Scientific cloud computing: Early definition and experience," in HPCC'08, 2008.

[5] M. C. Gonzalez, C. A. Hidalgo, and A.-L. Barabasi, "Understanding individual human mobility patterns," Nature, vol. 453, pp. 779-782, June 2008.

[6] E. ONeill, V. Kostakos, T. Kindberg, A. Fatah gen. Schiek, A. Penn, D. Stanton Fraser, and T. Jones, "Instrumenting the city: developing methods for observing and understanding the digital cityscape," in UbiComp '06, 2006.

[7] L. McNamara, C. Mascolo, and L. Capra, "Media sharing based on colocation prediction in urban transport," in MobiCom '08, 2008.

[8] J. Peltotalo, J. Harju, M. Saukko, L. Vaatamoinen, I. Bouazizi, I. Curcio, and J. van Gassel, "A real-time peer-to-peer streaming system for mobile networking environment," in INFOCOM Workshops 2009, IEEE.

[9] S. Xie, B. Li, and G. Keung, "The peer-to-peer live video streaming for handheld devices," in CCNC'08., 10-12 2008. 
[10] J. Akkanen, O. Karonen, and J. Porio, "Peer-to-peer video streaming on mobile phones," in CCNC'08., 2008.

[11] M. J. Osborne and A. Rubinstein, A Course in Game Theory. MIT Press, 1994.

[12] S. Boyd and L. Vandenberghe, Convex optimization. Cambridge Univ Press, 2004.

[13] N. Eagle, A. Pentland, and D. Lazer, "Inferring social network structure using mobile phone data," PNAS, 2007.

[14] G. M. J. G. E. P. Box and G. C. Reinsel, Time Series Analysis: Forecasting and Control (3rd Edition). Prentice Hall, 1994. 